\title{
The generation of antibody diversity through somatic hypermutation and class switch recombination
}

\author{
Ziqiang Li, ${ }^{1}$ Caroline J. Woo, ${ }^{1}$ Maria D. Iglesias-Ussel, Diana Ronai, and Matthew D. Scharff ${ }^{\mathbf{2}}$ \\ Department of Cell Biology, Albert Einstein College of Medicine, New York, New York 10461, USA
}

Antibody-mediated immunity is critical to the resistance of vertebrate species to pathogenic organisms. Although low-affinity immunoglobin (Ig) M antibodies circulate in the blood prior to encountering pathogens, high-affinity IgG and IgA antibodies are required to inactivate toxins, neutralize viruses, and promote the clearance of microorganisms. Individuals, such as those with hyper-IgM syndrome (HIGM), who lack the ability to make such high-affinity IgG and IgA antibodies, are unable to combat bacterial and viral infections and usually die at a young age (Revy et al. 2000; Imai et al. 2003). Prior to exposure to antigen, the initial generation of a diverse antibody repertoire is achieved early in B-lymphocyte development by the successful rearrangement of the V, D, and J gene segments to produce B cells, each of which makes a unique Ig heavy- and light-chain variable (V) region (Fig. 1A; Tonegawa 1983). These V regions encode the antigen binding sites of antibodies that are then expressed on the surface of a B lymphocyte and its clonal progeny. Following specific antigen recognition by its cognate B lymphocyte and costimulation by helper $\mathrm{T}$ lymphocytes, the B lymphocyte enters the germinal center of peripheral lymphoid organs to become a centroblast B cell. There, a second wave of antibody diversification occurs through somatic hypermutation (SHM) and/or gene conversion (GC) of the $\mathrm{V}$ region to generate high-affinity antigen binding sites (Fig. 1B; MacLennan 1994). SHM is the predominant mechanism in mice and humans, whereas GC occurs in chickens and some other species (Weill and Reynaud 1996). In the same centroblast $\mathrm{B}$ cell, the heavy-chain $\mathrm{V}$ regions encoding the antigen binding sites are rearranged down the chromosome through class switch recombination (CSR) so that they can be expressed with one of the constant (C) region genes to carry out many different effector functions and be distributed throughout the body (Fig. 1B; Manis et al. 2002b). SHM and CSR are largely targeted to the Ig genes,

\footnotetext{
${ }^{1}$ These authors contributed equally to this review.

${ }^{2}$ Corresponding author.

E-MAIL scharff@aecom.yu.edu; FAX (718) 430-8574.

Article and publication are at http://www.genesdev.org/cgi/doi/10.1101/ gad.1161904.
}

but their targeting to other genes causes many of the B-cell lymphomas in mice and humans (Pasqualucci et al. 2001). The biochemical mechanism and regulation of SHM and CSR are the topic of this review.

\section{The characteristics of SHM}

In mice and humans, SHM occurs at rates of $10^{-5}$ to $10^{-3}$ mutations per base pair per generation which is $\sim 1$ million-fold higher than the spontaneous rate of mutation in most other genes (Rajewsky et al. 1987). The mutations are mainly single base substitutions, with occasional insertions and deletions. These mutations are targeted to the $\mathrm{V}$ region, beginning $\sim 150$ to $200 \mathrm{bp}$ downstream of the promoter, and extend $\sim 1.5-\mathrm{kb}$ further downstream, ending before the intronic enhancer $(\mathrm{E} \mu)$ and sparing the C region (Fig. 1B; for review, see Rada and Milstein 2001). Although mutations occur throughout the rearranged $\mathrm{V}$ regions and its immediate flanking sequences, there is preferential targeting to $\mathrm{WRCY}(\mathrm{W}=\mathrm{A}$ or $\mathrm{T}$, $\mathrm{R}=\mathrm{A}$ or $\mathrm{G}, \mathrm{C}, \mathrm{Y}=\mathrm{T}$ or $\mathrm{C}$ ) and WA motifs that are therefore referred to as hot spots. In any particular $\mathrm{V}$ region, some potential hot spots are targeted and others are not, suggesting that other local sequences or higher-order structures may also influence the targeting of mutations (Rogozin and Kolchanov 1992; Kinoshita and Honjo 2001). In addition, transition mutations arise more frequently than do transversions.

\section{Characteristics of class switch recombination}

In CSR, the $\mathrm{C} \mu$ region is replaced by downstream $\mathrm{C} \gamma, \mathrm{C} \varepsilon$, or $\mathrm{C} \alpha$ segments to generate IgG, IgE, and IgA antibodies, respectively. This process is mediated by an intrachromosomal recombinational event between the switch (S) region of the $\mathrm{C} \mu$ region $(\mathrm{S} \mu)$ and one of the downstream $\mathrm{S}$ regions (Fig. 2). These S regions are located immediately upstream of each of the $\mathrm{C}$ regions (except for $\delta$; Fig. 2A) and contain imperfect G-C-rich pentameric repeats that serve as the donors and recipients for a recombinational process that is region specific but not sequence specific (for review, see Stavnezer 2000; Kenter 2003). 
Li et al.

Figure 1. DNA transactions that contribute to the generation of antibody diversity in mammalian B lymphocytes. (A) V(D)J rearrangement. The genomic organization of the murine germline Ig heavy-chain locus is shown on the top diagram. One diversity (D) segment (purple rectangles) and one joining segment (yellow rectangles) rearrange to form DJ segment (middle diagram) that subsequently recombines with a variable (V) segment (orange rectangles) to form a VDJ segment (bottom diagram). Transcription of this locus is regulated by the intronic enhancer, E $\mu$ (gray oval). (B) SHM, GC and CSR. Ig heavy-chain (top) and $\kappa$ light-chain (bottom) genes that have undergone $\mathrm{V}(\mathrm{D}) \mathrm{J}$ rearrangement are shown, with the symbols as in $A$. Enhancers that regulate the expression of these genes are shown as gray ovals.
A

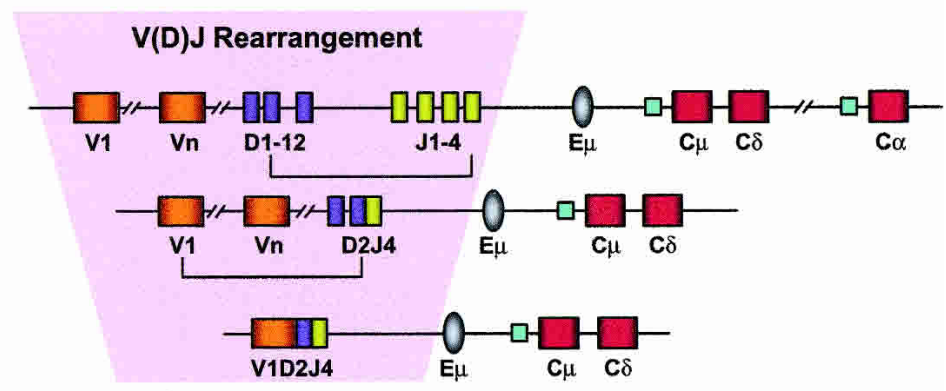

B

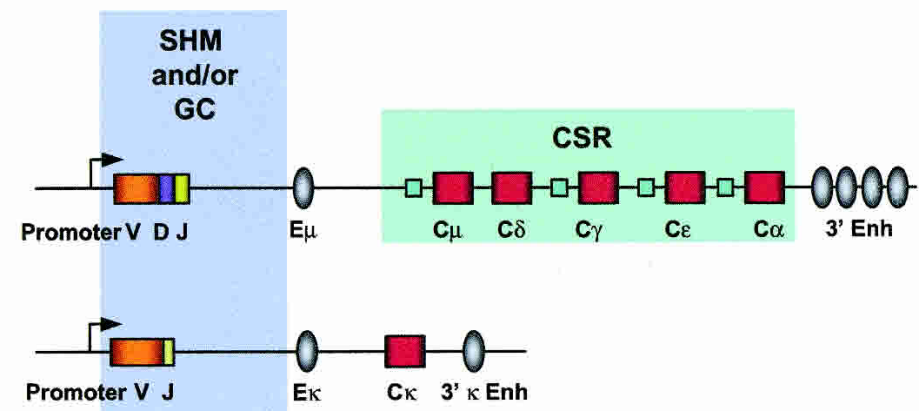

CSR requires the production of sterile transcripts from a promoter located just $5^{\prime}$ to each of the $\mathrm{S}$ regions (Fig. 2B). Lymphokines produced by different subsets of helper $\mathrm{T}$ cells that interact with the B cells signal the activation of the promoter for individual $S$ regions, thus instructing the B cells to transcribe the sterile transcripts for $S \mu$ and a particular downstream $S$ region and subsequently to juxtapose that $\mathrm{C}$ region to $\mathrm{S} \mu$ region (Fig. $2 \mathrm{~B}, \mathrm{C}$; for review, see Manis et al. 2002b; Kenter 2003). The sterile transcript is thought to form a RNA-DNA hybrid with the template strand, and this results in the exposure of a region of single-stranded DNA (ssDNA) on the nontemplate strand that may be as long as $1 \mathrm{~kb}$ (Shinkura et al. 2003; Yu et al. 2003). This structure is called an R loop (Fig. 2B) and presumably makes the $S$ region accessible to mutation. The subsequent rearrangement thus juxtaposes the heavy-chain $\mathrm{V}$ region that may have undergone SHM to one or another of the downstream $\mathrm{C}$ regions (Fig. 2D), allowing each antigen binding site to mediate many different effector functions. It is important to note that point mutations with the similar characteristics to those seen in $\mathrm{V}$ region SHM are found in the donor and recipient $\mathrm{S}$ regions flanking the site of recombination (ReinaSan-Martin et al. 2003).

\section{Molecules involved in SHM and CSR}

Even though SHM and CSR occur in B cells at the same stage of differentiation, they appear to be very different biochemical processes. There are IgM antibodies expressing V regions that have undergone SHM, as well as IgG and IgA antibodies with no somatic mutations, suggesting that SHM and CSR are independent processes mediated by completely different sets of enzymes. This was supported by the finding that CSR requires $\mathrm{Ku}$, DNA-PK ${ }_{\mathrm{cs}}, \mathrm{H} 2 \mathrm{AX}$, and perhaps other enzymes involved in repairing double-stranded DNA breaks, whereas these molecules are dispensable for SHM (Bemark et al. 2000; Manis et al. 2002a; Reina-San-Martin et al. 2003). However, in the last few years, the discovery of additional molecules that are responsible for SHM and CSR has revealed surprising connections between these two processes.

\section{Activation-induced cytidine deaminase}

The discovery of activation-induced cytidine deaminase (AID), and the demonstration that it is required for SHM, GC, and CSR triggered many of the recent advances in this field (Muramatsu et al. 1999). AID expression is restricted to centroblast B cells, and mice deficient in AID are completely defective in SHM and CSR (Muramatsu et al. 2000). Patients with HIGM2 harboring inactivating AID mutations are unable to undergo SHM and CSR (Revy et al. 2000), and chicken DT-40 cells lacking AID are unable to carry out GC (Harris et al. 2002). Ectopic expression of AID causes SHM in B cells at an incorrect stage of differentiation (Martin et al. 2002), in non-B cells (Martin and Scharff 2002b; Yoshikawa et al. 2002), and in Escherichia coli (Petersen-Mahrt et al. 2002), suggesting that AID is the only B cell-specific protein required for SHM and CSR (Martin and Scharff 2002a).

AID is homologous and linked to apolipoprotein B (apoB) mRNA-editing enzyme catalytic polypeptide 1 (APOBEC-1) that deaminates cytidine 6666 to a uracil in the apoB mRNA to generate a premature stop codon and a smaller protein that binds a different receptor than the full length protein (Navaratnam et al. 1993). This origi- 


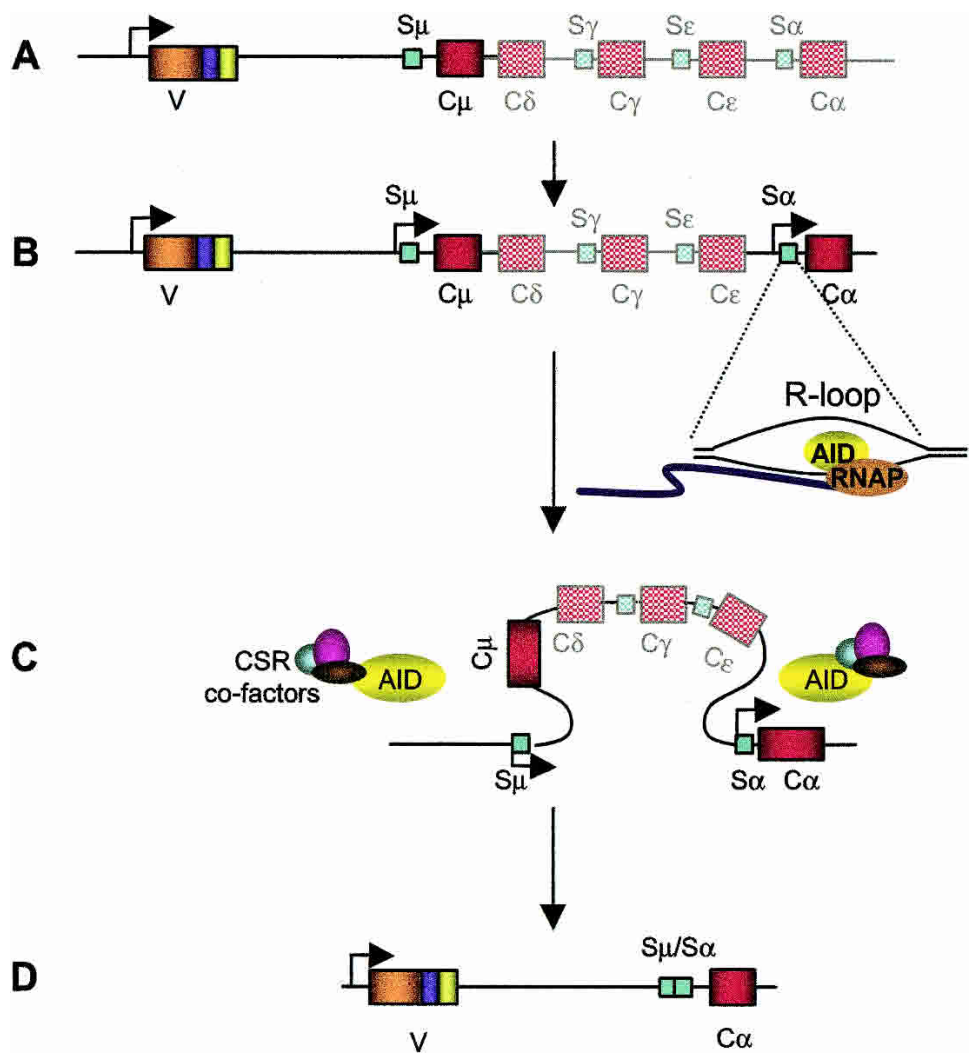

Figure 2. Model of class switch recombination. $(A)$ The genomic organization of the rearranged heavychain Ig locus is shown with the same symbols as in Fig. 1. (B) During CSR, promoters for sterile transcripts are active (shown as arrows upstream of switch regions). R-loop forms in which RNA (blue line) remains bound to DNA (black lines) after the RNA polymerase (RNAP) has transcribed the region. $(C)$ The region between $\mathrm{S} \mu$ and $\mathrm{S} \alpha$ is deleted. $(D) \mathrm{C} \mu$ is replaced by $\mathrm{C} \alpha$. nally led to the suggestion that AID was editing mRNA encoding an endonuclease that acted on $\mathrm{V}$ regions to mediate SHM and GC and on S regions to trigger CSR (Kinoshita and Honjo 2001). Although the catalytic site of AID is homologous to the catalytic site of RNA-editing enzymes, it seemed possible from the outset that it was working directly on DNA (Poltoratsky et al. 2000), and evidence rapidly accumulated suggesting that this was true. In particular, it seemed unlikely that there would be a target mRNA to be edited in $\mathrm{CHO}$ and 3T3 cells and in bacteria where AID is not normally expressed (Martin and Scharff 2002b; Petersen-Mahrt et al. 2002; Yoshikawa et al. 2002). This, coupled with the findings that an inhibitor of uracil $N$-glycosylase (UNG) blocked GC in DT-40 cells (Di Noia and Neuberger 2002) and that mice lacking UNG had a perturbed SHM spectra and a decrease in CSR (Rada et al. 2002), made it a virtual certainty that DNA is the substrate for AID.

This DNA deamination model has now been supported biochemically. By using semipurified AID in a cell free system, Bransteitter et al. (2003) showed that GST-tagged AID isolated from insect cells was able to deaminate $\mathrm{dC}$ to $\mathrm{dU}$ in ssDNA but not in doublestranded DNA, RNA, or DNA-RNA hybrids. In addition, Pham et al. (2003) used this semipurified AID in a phage-based reporter system to generate a large database of in vitro mutations and showed that AID preferentially targeted the dC in WRC motifs. Furthermore, by using T7 polymerase-dependent system, these investigators found that most of the mutations were on the nontran- scribed strand, although in vivo both of the transcribed and nontranscribed strands are targeted (Milstein et al. 1998). By using extracts from cells overexpressing AID, Chaudhuri et al. (2003) also observed dC deamination activity of $\mathrm{C}$ in hot spot motifs only on ssDNA. Similar findings were also observed by others using AID made in E. coli (Dickerson et al. 2003; Sohail et al. 2003), although the AID made from bacteria did not preferentially target hot spot motifs. The reason for this discrepancy is unclear.

It is unclear whether there are other molecules associated with AID that are responsible for its preferential targeting to the Ig V region for SHM and to $\mathrm{S}$ regions for CSR. However, recent studies showed that mutations or deletions in the C-terminal 8 to 17 amino acids of AID do not trigger CSR, even though these mutant AID molecules can be targeted to the $S$ region to generate point mutations and retain GC activity in DT-40 cells and mutagenic activity in E. coli and fibroblasts (Ta et al. 2003). These results suggest that different domains of AID recruit factors that differentially mediate the outcomes of SHM and CSR.

\section{Uracil N-glycosylase}

As already noted above, UNG, a uracil DNA glycosylase, plays an important role in SHM and CSR, presumably by removing uracil generated by AID deamination. Deletion of UNG in E. coli that overexpresses AID and inhibition of UNG in DT-40 cells result in an increase in 
transition mutations on G-C base pairs (Petersen-Mahrt et al. 2002). UNG also plays an important role in CSR. Mice deficient in UNG had fivefold to 10-fold reduction of switching from IgM to IgG3 and $~ 10$-fold reduction of switching to IgG1, suggesting that removing uracil to generate abasic sites is an important pathway in CSR (Rada et al. 2002). Patients with homozygous recessive mutations in the active site of UNG exhibit a high level of IgM but very low levels of IgG or IgA in their sera, and their B cells have a greatly reduced ability to carry out CSR after cytokine stimulation in vitro (Imai et al. 2003). Although there are other uracil DNA glycosylases in animal cells, such as MBD4, TDG, and SMUG, the findings in UNG-deficient mice and humans suggest that UNG is the major glycosylase involved in SHM and CSR. This has been supported by a study in which neither process is perturbed in MBD4-deficient mice (Bardwell et al. 2003).

\section{Mismatch repair proteins}

Mismatch repair (MMR) normally maintains genomic integrity (Kolodner 1996). In humans and mice, the MSH2-MSH6 heterodimer recognizes single or very short base-pair mismatches, whereas the MSH2-MSH3 heterodimer recognizes larger mismatches or insertions or loops (Buermeyer et al. 1999; Wei et al. 2002). The recognition of a mismatch is followed by the recruitment of downstream mismatch repair proteins-such as MLH1, PMS2, PCNA, Exonuclease 1 (EXO1), and unknown endonuclease(s) - to excise the mismatched bases and DNA polymerase(s) to fill the gap and restore the DNA sequence (Kolodner 1996). Mice deficient in MMR proteins exhibit microsatellite instability and cancerprone phenotype (Wei et al. 2002).

MMR eliminates mutations that arise spontaneously in the genome. Mice or cell lines deficient in MMR proteins exhibit an increased level of spontaneous mutations (Wei et al. 2002). In contrast, mice deficient in MSH2 or MSH6 show a decreased frequency of SHM (for review, see Martin and Scharff 2002a). Very importantly, the spectrum of SHM in mice deficient in $\mathrm{MSH} 2$ or MSH6 is different from wild-type mice; that is, there are very few mutations in A-T and the majority of mutations are G-to-A or C-to-T transitions at G-C base pairs in WRCY motifs. These findings led to the proposal that SHM has two phases; that is, in the first phase, AID and UNG generate mutations on G-C pairs, and in the second phase, MMR is recruited to exercise the DNA containing the mismatches and resynthesize the excised DNA with the help of error-prone polymerases such as pol $\eta$. This results in additional mutations in $A$ and $T$, as well as C, that are not in hotspots (Rada et al. 1998; Zeng et al. 2001).

In mice deficient in MLH1 or PMS2, two proteins involved in the later stages of mismatch repair, the changes in the mutation frequency and in the spectra of mutations are less dramatic than in MSH2-deficient mice, and in some experiments it was difficult to distinguish the mutant mice from their wild-type littermate controls, although there does appear to be a slight increase mutations at G-C pairs (for review, see Martin and Scharff 2002a). Although these results cannot be easily explained, it is possible that the role of MLH1 and/or PMS2 in SHM is different from that in MMR (Schrader et al. 2003) or that there is functional redundancy for MLH1 and PMS2 (Arakawa et al. 2002).

MMR proteins are also involved in CSR. Studies with MSH2-deficient mice have reported a twofold to 10-fold reduction in isotype switching (Ehrenstein and Neuberger 1999; Schrader et al. 1999), suggesting that the MSH2 protein is needed to carry out efficient CSR. In addition, mice carrying a mutation in the MSH2 ATPase domain have a deficiency in switching, suggesting the ATPase activity of MSH2 is essential for efficient CSR (Martin et al. 2003). Impaired CSR is also observed in MSH6-deficient mice but not in MSH3-deficient mice ( $\mathrm{Z}$. Li and M.D. Scharff, unpubl.), suggesting that, as with SHM, it is the MSH2-MSH6 complex that is important for SHM and efficient CSR. PMS2- or MLH1-deficient mice exhibit approximately two to fourfold reduction of switching (Schrader et al. 1999; Ehrenstein et al. 2001).

Both MMR deficiency and UNG deficiency result in significant defects in CSR, although the phenotype of UNG deficiency may be stronger than that of MMR deficiency (Rada et al. 2002; Imai et al. 2003). It is difficult to envision that the UNG pathway is dominant over the MMR pathway or vice versa, if they are two independent pathways in CSR. It is therefore reasonable to postulate that MMR can recognize the G-abasic mismatches after UNG removes $U$ triggering CSR.

\section{Error-prone DNA polymerases}

Early explanations of the generation of antibody diversity invoked error-prone polymerases (Brenner and Milstein 1966), and when the mammalian homologs of the bacterial translesional polymerases were shown to have low fidelities, it was suggested that they might be involved in SHM (Goodman and Tippin 2000). As already noted, this has been confirmed by the finding that patients with deficiencies in pol $\eta$ have a decreased frequency in mutations at $A$ and $T$ (Zeng et al. 2001). Interference with pol $\zeta$ in mice and in a Burkitt's lymphoma cell line decreases in the rate of SHM (Diaz et al. 2001; Zan et al. 2001). In addition, Burkitt's cells in which pol ı has been inactivated have an $\sim 75 \%$ reduction of SHM frequency (Faili et al. 2002a). However, mice deficient in pol $\iota$ do not change their rate and spectrum of mutation (McDonald et al. 2003). The difference between these two results is difficult to reconcile, and further studies are required to fully understand the roles of these polymerases in SHM and to determine if they play any role in CSR.

\section{Nonhomologous end joining}

Nonhomologous end joining (NHEJ) plays an essential role during $\mathrm{V}(\mathrm{D}) \mathrm{J}$ recombination and in the repair of 
double-stranded DNA breaks. Ku70, Ku80, DNA-PKcs, or H2AX- deficient mice showed an impaired CSR activity (Manis et al. 2002b; Reina-San-Martin et al. 2003), whereas they are normal for SHM (Bemark et al. 2000; Reina-San-Martin et al. 2003), suggesting that ligating of two double-stranded break ends is not a prominent process during SHM. The fact that NHEJ is important for CSR but not for SHM supports the idea that AID-initiated G-U mismatches are resolved differently during SHM and CSR (for review, see Li et al. 2003). Although H2AX-deficient mice exhibit normal SHM (Reina-SanMartin et al. 2003), in hypermutating Burkitt's lymphoma cell lines $\gamma$-H2AX is associated with the $\mathrm{V}$ region but not C region (Woo et al. 2003; Zan et al. 2003). This suggests that double-stranded breaks may arise during SHM but are not required for that process (Faili et al. 2002b; Papavasiliou and Schatz 2002), whereas they are essential for repairing the double-stranded DNA breaks that are a critical part of CSR.

\section{Model for the production of mutations in both SHM and CSR}

The recent explosion of information on molecules responsible for SHM and CSR described above has led to a general model (Poltoratsky et al. 2000; Petersen-Mahrt et al. 2002) that incorporates the findings described above and is used by many in the field to explain the initiation of both processes (Fig. 3). AID is only expressed in centroblast B cells, hence the restriction of SHM and CSR to this stage of B-cell differentiation, and the regulation of these processes depends upon the transcriptional activation of AID. Newly synthesized AID may associate with other molecules or undergo posttranslational modifications such as phosphorylation so that it can be transported from the cytoplasm to the nucleus. Although AID has a putative bipartite nuclear localization motif, it is not yet clear whether this is functional and sufficient, as well as whether AID needs to be chaperoned into the nucleus. Once it is transported into the nucleus, AID interacts with ssDNA in the $\mathrm{V}$ and the $\mathrm{S}$ regions and deaminates $\mathrm{dC}$ to $\mathrm{dU}$ to produce $\mathrm{G}-\mathrm{U}$ mismatches. As diagramed in Figure 3, the dU created by AID may subsequently be replicated to produce a C-to-T mutation or G-to-A mutation on the other strand (phase I A) or removed by UNG to create an abasic site. The abasic site can subsequently be converted to a single-stranded nick by AP-endonuclease and then repaired by base excision repair without generating mutations, or it can be bypassed by error-prone DNA polymerase(s) to generate mutations (phase I B), recognized by MSH2-MSH6, and then excised and replaced by resynthesis with errorprone polymerases that will create additional mutations including mutations on A-T base pairs (phase II). It is unclear whether these processes compete with each other in a random fashion or whether they are coordinated and regulated by other factors such as the cell cycle-mediated levels of expression of the relevant proteins. In addition, there is some indirect evidence that
AID introduces many more lesions than are ultimately fixed as point mutations in the $\mathrm{V}$ region and that many of the AID-induced mutations are repaired by homologous recombination in late $S$ and $G_{2}$ (Sale and Neuberger 1998; Faili et al. 2002b). A role for homologous recombination has also been invoked by recent studies of SHM in a Burkitt's lymphoma cell line (Zan et al. 2003).

After $\mathrm{dU}$ is introduced into the $\mathrm{S}$ regions, there is the additional requirement for double-stranded DNA breaks to allow for $\mathrm{S}$ recombination. The double-stranded DNA breaks in the $S$ region may be staggered and could be the result of many single-stranded breaks resulting from the activities of AID, UNG, MMR, and AP-endonuclease, or they may be created by some as yet unidentified mechanism. Once the double-stranded DNA breaks are created and processed in the $S$ region, they can be repaired by NHEJ mechanism or can be resolved by other DNA repair mechanisms such as homologous recombination. Although this model for the mutations that occur in the $\mathrm{V}$ and $\mathrm{S}$ regions explains many of the observations described in the previous sections, it is incomplete and could even be incorrect because there are still many unresolved questions and controversial issues, some of which will now be discussed.

\section{Is MMR playing a direct or indirect role in SHM and CSR?}

The fact that MSH2-deficient mice change the spectra of SHM, the frequency of CSR, and the characteristics of the recombination sites in CSR suggests MMR plays a direct and important role in both of these processes (Martin and Scharff 2002a). However, some investigators have suggested that the defects in these processes in the MMR-deficient mice led to an arrest of B-cell development due the genomic instability in rapidly dividing $B$ cells or to the loss of apoptotic or other signals (Frey et al. 1998; Vora et al. 1999). This concern also arises from the observation that the defects in SHM that have been observed in $\mathrm{MSH}^{-/-}$or $\mathrm{MSH}^{-/-}$mice are more pronounced than are the defects in MLH1- or PMS2-deficient mice, even though the latter act downstream in the MMR process and would be expected to lead to the same defects (for review, see Martin and Scharff 2002a). This enigma is compounded by the observation that the $S$ junctions look different in the mice with deficiencies in different MMR proteins. It was expected that the sequences of these sites of recombination would reveal the biochemical processes that had occurred (Stavnezer 2000). The Su-S $\gamma 3$ junctions from splenic B cells of MSH2-deficient mice stimulated to switch in vitro showed shorter microhomologies, more blunt junctions, and more insertions than do wild-type mice (Schrader et al. 2002). Unexpectedly, PMS2- or MLH1-deficient mice exhibited more long microhomologies in the $\mathrm{S}$ junctions than did wild-type mice (Ehrenstein et al. 2001; Schrader et al. 2002). Furthermore, doubly-deficient MSH2/MLH1 mice exhibit switching junctions similar to that of MLH1- but not of MSH2-deficient mice, although dou- 
Li et al.
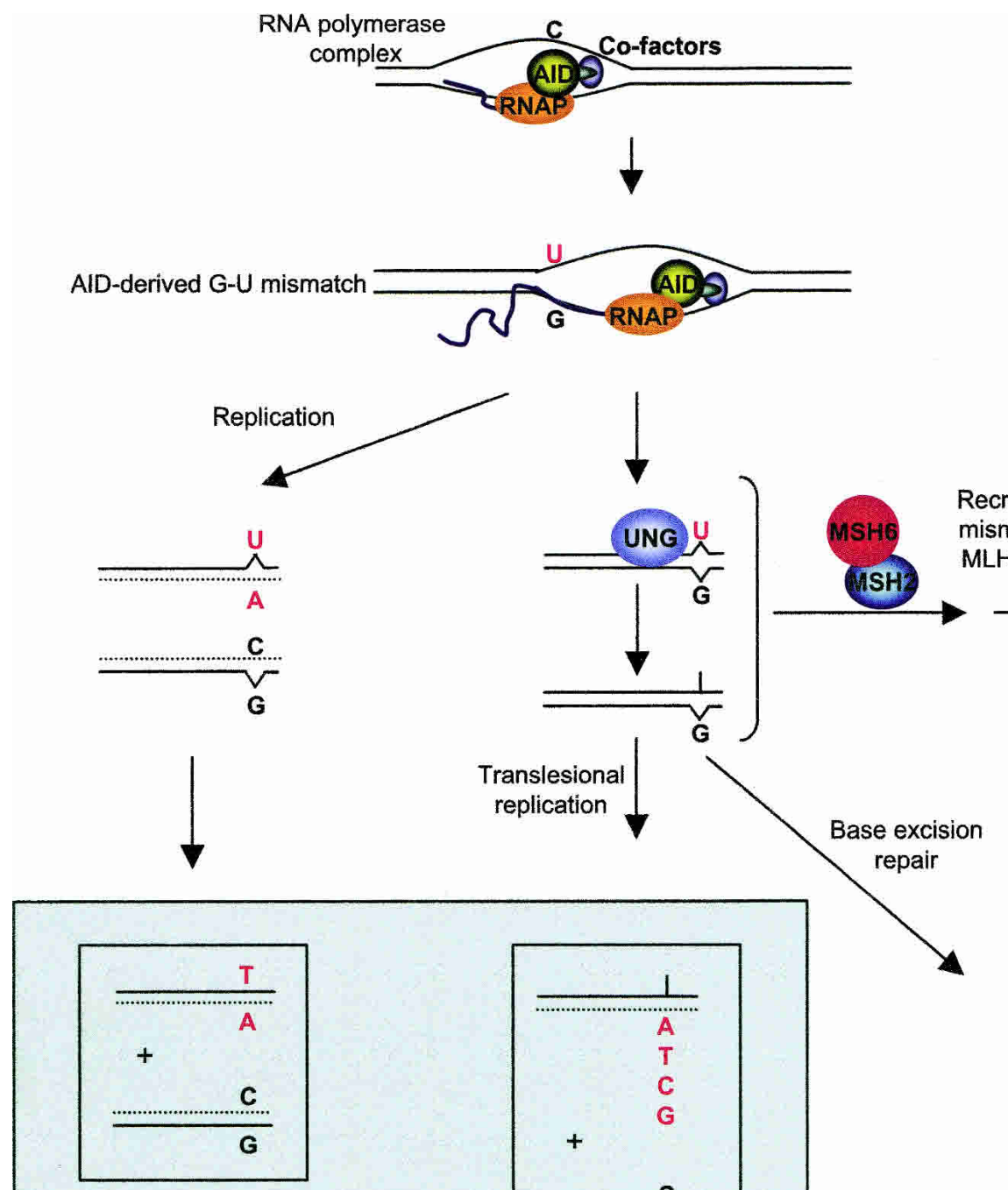

A
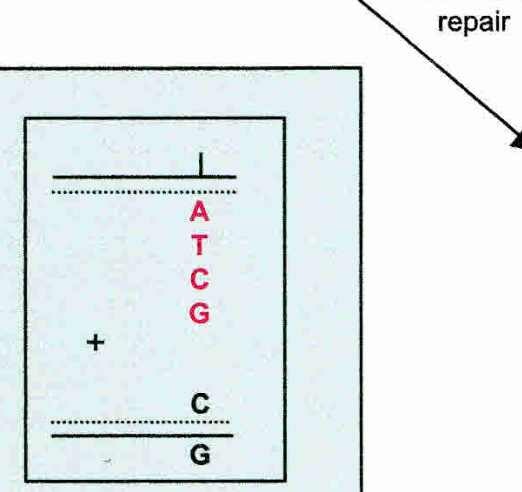

\section{PHASE I}

B

Figure 3. Model of somatic hypermutation. During RNA polymerase (RNAP, orange oval)-mediated transcription, AID (yellow oval) and cofactors (blue ovals) deaminate cytosines $(\mathrm{C})$ on the single-stranded DNA of the transcription bubble to generate uracil (U). The result of this reaction is a G-U mismatch which can be resolved as follows: (phase I, A) G-U mismatches undergo DNA replication and result in C-to-T mutation. (B) UNG removes uracil, and the resulting abasic site is either processed by base excision repair in an error-free fashion or replicated to any base. (phase II) Mismatch repair (MMR) proteins MSH2 and MSH6 (blue and red ovals) bind to G-U mismatches or G-abasic mismatches and subsequently recruit additional MMR proteins as well as error-prone polymerases to generate mutations on $\mathrm{A} / \mathrm{T}$ base pairs.

bly and singly deficient mice exhibit a similar extent of reduction in switching to different isotypes compared with the wild-type controls (Schrader et al. 2003). What is especially interesting is that ATPase-deficient MSH2 mice exhibit a mixed phenotype of switching junctions; that is, they have longer microhomologies, such as PMS2- or MLH1-deficient mice, and they have more insertions, such as the MSH2-deficient mice (Martin et al. 2003). It is difficult to explain the meaning of different sized microhomologies and insertions in $S$ junctions in the mice lacking various MMR proteins. Because the exact mechanism of CSR is unclear at this point, additional in vivo and in vitro experiments will be required to clarify this.

Despite these uncertainties, it is hard to envision how these differences in the patterns of mutation in SHM and the characteristics of the $S$ junctions could arise as the result of some nonspecific arrest in B-cell development, so these observations seem to support a direct role for MMR in SHM and CSR. A direct role for MMR in SHM 
is further supported by our recent observation that chromatin immunoprecipitation (ChIP) with antibodies against Exo1 and Mlh1 reveal that they are physically associated with $\mathrm{V}$ region but not the $\mathrm{C}$ region in BL2 cells undergoing SHM (P.D. Bardwell and M.D. Scharff, unpubl.). Furthermore, MSH2 ATPase mutant mice, which are deficient in mismatch repair but retain the ability to signal for apoptosis, exhibit the characteristics of SHM and CSR similar to those of MSH2 complete knock-out mice, suggesting that it is MMR activity per se that is involved in antibody diversification process rather than triggering apoptosis (Martin et al. 2003).

\section{How is AID targeted to the $\mathrm{V}$ and $\mathrm{S}$ regions but not to other genes?}

What is the role of transcription in SHM and CSR?

The importance of the promoter in determining the target of mutation has been confirmed in many ways, including experiments in which the $\mathrm{C}$ region underwent SHM when the V region promoter was placed just upstream of it (Peters and Storb 1996). In addition, there are a number of experiments showing that the rate of $\mathrm{V}$ region mutation is roughly proportional to the rate of transcription (Fukita et al. 1998; Bachl et al. 2001; Ramiro et al. 2003). Although there were many possible explanations for this relationship to transcription, the recent biochemical finding that AID deaminates $\mathrm{dC}$ in ssDNA in cell-free systems in the absence of transcription shows that transcription per se and RNA polymerase II are not required for AID activity in vitro. Furthermore, mutation is targeted to ssDNA regions resulting from transcription by T7 RNA polymerase, confirming that RNA polymerase II is not required in vitro. Rather, these studies suggest that transcription is required because it creates ssDNA regions in the form of transcription bubbles in the $\mathrm{V}$ region (Bransteitter et al. 2003; Chaudhuri et al. 2003) and R loops in the $S$ region (Shinkura et al. 2003; $\mathrm{Yu}$ et al. 2003). If AID binds strongly to ssDNA in vivo and behaves in a processive manner (Pham et al. 2003), it will be interesting to learn more about the relationship between AID, RNA polymerase II, and the moving transcription bubble in the $\mathrm{V}$ region and in the $\mathrm{R}$ loop in the $S$ region. Furthermore, the biochemical studies show a predominance of mutations in the nontranscribed strand, so we still have to learn how the mutations in the template strand arise (Chaudhuri et al. 2003; Dickerson et al. 2003; Pham et al. 2003; Ramiro et al. 2003; Sohail et al. 2003).

\section{Are there cis-acting sequences that target AID for mutations?}

The analyses of many Ig transgenes in vivo revealed that different promoters could substitute for the $\mathrm{V}$ region promoter and SHM would still be targeted to the $\mathrm{V}$ region (Betz et al. 1994; Tumas-Brundage and Manser 1997). In cultured B cells, viral promoter-enhancers could substitute for E $\mu$ without affecting the rate or characteristics of mutation (Bachl and Wabl 1996; Lin et al. 1998). Interestingly, the $3^{\prime}$ enhancer of the light-chain is also required for high rates of SHM in the light-chain $\mathrm{V}$ region in vivo (Sharpe et al. 1990; Tumas-Brundage et al. 1997), although the heavy-chain 3' enhancer is not required for heavy-chain $\mathrm{V}$ region mutation (Tumas-Brundage et al. 1997; Morvan et al. 2003) but is required for CSR (for review, see Manis et al. 2002b). Because these transcriptional regulatory elements do not appear to contain particular sequence motifs required for the targeting of SHM, the V region coding exon was examined for cisacting sequences by replacing it with all sorts of genetic elements, including genes from bacteria and Drosophila, in transgenic mice (Azuma et al. 1993; Yelamos et al. 1995; Tumas-Brundage et al. 1996). In general, these foreign genes were targeted for the same sorts of mutations as the $\mathrm{V}$ region, suggesting that the $\mathrm{V}$ region exon also does not contain any particular cis-acting motifs required for targeting of SHM. The finding that non-Ig genes could be targeted by AID for mutation in B cells and in non-B cells (Martin and Scharff 2002b; Yoshikawa et al. 2002) has added to the uncertainty about whether there are particular DNA binding proteins such as specific transcription factors required for SHM. The realization that SHM was proportional to the rate of transcription and the observation that some oncogenes in B-cell malignancies had a pattern of mutation that resembled SHM had already led to an examination of whether other highly transcribed genes in centroblast B cells were undergoing SHM. This appears to be true for Bcl6 in human cells but not in mice (Pasqualucci et al. 1998; Shen et al. 1998). It was also found that $\mathrm{mb1}$ and B29, highly expressed genes that encode proteins associated with IgM in surface of B cells, had mutations with the characteristics of SHM in normal and malignant human cells (Gordon et al. 2003). However, mutations are not found in all highly transcribed genes in B cells (Shen et al. 2000). This suggests that there are cis-acting sequences that play a role in targeting AID to particular genes. Recently, Michael et al. (2003) reported that an E-box motif increases the rate of mutation of a synthetic transgene in mice. In all of these studies, there are still issues about whether the rates of SHM are comparable to those that occur naturally, whether the observed mutations are really AID-dependent, and whether these different genes really have similar rates of transcription. In addition, it is likely that the overexpression of AID will eliminate the need to have specific targeting mechanisms, which could explain some but not all of the observations of mutations in non-Ig genes (Woo et al. 2003).

\section{Does chromatin structure play a role in the targeting of SHM and CSR?}

Alt and his colleagues proposed that trans-acting proteins were targeted to the V region for SHM and the S regions for CSR by increasing the accessibility of the relevant DNA domains, and they have recently sug- 
gested that changes in chromatin structure are associated with expression and rearrangement of the Ig genes (Mostoslavsky et al. 2003). In addition to the important role of histone modifications in modulating $\mathrm{V}(\mathrm{D}) \mathrm{J}$ recombination at the IgH locus (for review, see Schlissel 2000), such modifications are also critical during CSR in centroblasts. In response to specific cytokine stimuli, there is an increase in the accessibility of the $S$ region that leads to germline transcription of the $S$ and $C$ region (Fig. 2; Stavnezer 2000). This is associated with increases in the DNase I hypersensitivity of the specific $S$ regions that will subsequently undergo CSR (for review, see Stavnezer 1996). In at least some instances, these events may be associated with histone deacetylation of a microdomain within Ig gene because CSR to IgA can be suppressed by the DNA-binding protein, late SV40 factor (LSF), that recruits histone deacetylases and a corepressor to the $S$ region (Drouin et al. 2002).

Because chromatin structure plays an important role in both $\mathrm{V}(\mathrm{D}) \mathrm{J}$ rearrangement and CSR, it seemed reasonable that it would also be important in restricting SHM to the Ig gene and in targeting it to the $\mathrm{V}$ region and not to the $\mathrm{C}$ region. We have examined this by using the BL2 Burkitt's lymphoma cell line that can be induced to undergo SHM by stimulation with anti-IgM and helper $\mathrm{T}$ cells. In these costimulated mutating cells, there was a fivefold to 10-fold increase in the acetylation of $\mathrm{H} 4$ and about three- to fivefold increase in the actylation of $\mathrm{H} 3$ associated with $\mathrm{V}$ region, but there was no increase in the acetylation of the histones associated with the $\mathrm{C}$ region or the promoter (Woo et al. 2003). In addition, the histone modifications arose independently of the AID-induced mutations. However, when AID was overexpressed or hyperacetylation of the $\mathrm{C}$ region was induced, the $\mathrm{C}$ region was also targeted for SHM. We interpret these findings to mean that the induction of AID and hyperacetylation of the histones associated with the $\mathrm{V}$ region are two independent events. Very high levels of AID disrupted the tightly controlled targeting to $\mathrm{V}$ regions and to hot spots. If the chromatin associated with the $\mathrm{C}$ region is hyperacetylated, normal levels of AID can gain access to it. We hypothesized that in vivo, just enough AID is expressed so that the chromatin modifications in the nucleosomes associated with the $\mathrm{V}$ region allow or recruit the selective targeting of mutation to the $\mathrm{V}$ region of the Ig gene (Woo et al. 2003).

\section{Future Direction}

SHM and CSR occur during a brief stage in B-cell development and are both triggered by AID that converts $\mathrm{dC}$ to $\mathrm{dU}$ in the $\mathrm{Ig} \mathrm{V}$ and $\mathrm{S}$ regions. The lesions that are generated by this mutation are resolved by different mechanisms depending whether they arise in the V region or in the S region of the heavy-chain Ig gene. Although many of the enzymes that are involved have been identified in the past few years, some of the mutational factors have yet to be discovered, and there is still much to be learned about how they are coordinated and tar- geted to the $\mathrm{V}$ and $\mathrm{S}$ regions. Furthermore, it is unclear why some highly expressed non-Ig genes are targeted for mutation, whereas others are not. This is important because the SHM of non-Ig genes leads to many of the B-cell malignancies.

\section{Acknowledgments}

We apologize to those whose work could not be cited due to space limitations. M.D.S. is supported by grants from the National Institutes of Health (NIH; CA 72649, CA102705, and AI 43937), and also by the Harry Eagle Chair provided by the National Women's Division of the Albert Einstein College of Medicine. Z.L. is a Cancer Research Institute Fellow. C.J.W. was supported by NIH-T32GM-07491 and NIH-9526-1482 grants. M.D.I.U. is a fellow of the Ministerio de Educacion, Cultura y Deporte (Spain). D.R. is supported by the Harry Eagle fellowship.

\section{References}

Arakawa, H., Hauschild, J., and Buerstedde, J.M. 2002. Requirement of the activation-induced deaminase (AID) gene for immunoglobulin gene conversion. Science 295: 13011306.

Azuma, T., Motoyama, N., Fields, L.E., and Loh, D.Y. 1993. Mutations of the chloramphenicol acetyl transferase transgene driven by the immunoglobulin promoter and intron enhancer. Int. Immunol. 5: 121-130.

Bachl, J. and Wabl, M. 1996. Enhancers of hypermutation. Immunogenetics 45: 59-64.

Bachl, J., Carlson, C., Gray-Schopfer, V., Dessing, M., and Olsson, C. 2001. Increased transcription levels induce higher mutation rates in a hypermutating cell line. J. Immunol. 166: 5051-5057.

Bardwell, P.D., Martin, A., Wong, E., Li, Z., Edelmann, W., and Scharff, M.D. 2003. Cutting edge: The G-U mismatch glycosylase methyl-CpG binding domain 4 is dispensable for somatic hypermutation and class switch recombination. $J$. Immunol. 170: 1620-1624.

Bemark, M., Sale, J.E., Kim, H.-J., Berek, C., Cosgrove, R.A., and Neuberger, M.S. 2000. Somatic hypermutation in the absence of DNA-PK or Rag1 activity. J. Exp. Med. 192: 15091514.

Betz, A.G., Milstein, C., Gonzalez-Fernandez, A., Pannell, R., Larson, T., and Neuberger, M.S. 1994. Elements regulating somatic hypermutation of an immunoglobulin $\kappa$ gene: Critical role for the intron enhancer/matrix attachment region. Cell 77: 239-248.

Bransteitter, R., Pham, P., Scharff, M.D., and Goodman, M.F. 2003. Activation-induced cytidine deaminase deaminates deoxycytidine on single-stranded DNA but requires the action of RNase. Proc. Nat1. Acad. Sci. 100: 4102-4107.

Brenner, S. and Milstein, C. 1966. Origin of antibody variation. Nature 211: 242-243.

Buermeyer, A.B., Deschenes, S.M., Baker, S.M., and Liskay, R.M. 1999. Mammalian DNA mismatch repair. Annu. Rev. Genet. 33: 533-564.

Chaudhuri, J., Tian, M., Khuong, C., Chua, K., Pinaud, E., and Alt, F.W. 2003. Transcription-targeted DNA deamination by the AID antibody diversification enzyme. Nature 422: 726730.

Diaz, M., Verkoczy, L.K., Flajnik, M.F., and Klinman, N.R. 
2001. Decreased frequency of somatic hypermutation and impaired affinity maturation but intact germinal center formation in mice expressing antisense RNA to DNA polymerase zeta. J. Immunol. 167: 327-335.

Dickerson, S.K., Market, E., Besmer, E., and Papavasiliou, F.N. 2003. AID mediates hypermutation by deaminating single stranded DNA. J. Exp. Med. 197: 1291-1296.

Di Noia, J. and Neuberger, M.S. 2002. Altering the pathway of immunoglobulin hypermutation by inhibiting uracil-DNA glycosylase. Nature 419: 43-48.

Drouin, E.E., Schrader, C.E., Stavnezer, J., and Hansen, U. 2002. The ubiquitously expressed DNA-binding protein late SV40 factor binds Ig switch regions and represses class switching to IgA. J. Immunol. 168: 2847-2856.

Ehrenstein, M.R. and Neuberger, M.S. 1999. Deficiency in msh2 affects the efficiency and local sequence specificity of immunoglobulin class-switch recombination: Parallels with somatic hypermutation. EMBO J. 18: 3484-3490.

Ehrenstein, M.R., Rada, C., Jones, A.M., Milstein, C., and Neuberger, M.S. 2001. Switch junction sequences in PMS2-deficient mice reveal a microhomology-mediated mechanism of Ig class switch recombination. Proc. Natl. Acad. Sci. 98: $14553-14558$.

Faili, A., Aoufouchi, S., Flatter, E., Gueranger, Q., Reynaud, C.A., and Weill, J.C. 2002a. Induction of somatic hypermutation in immunoglobulin genes is dependent on DNA polymerase iota. Nature 419: 944-947.

Faili, A., Aoufouchi, S., Gueranger, Q., Zober, C., Leon, A., Bertocci, B., Weill, J.C., and Reynaud, C.A. 2002b. AID-dependent somatic hypermutation occurs as a DNA single-strand event in the BL2 cell line. Nat. Immunol. 3: 815-821.

Frey, S., Bertocci, B., Delbos, F., Quint, L., Weill, J.C., and Reynaud, C.A. 1998. Mismatch repair deficiency interferes with the accumulation of mutations in chronically stimulated $\mathrm{B}$ cells and not with the hypermutation process. Immunity 9: $127-134$.

Fukita, Y., Jacobs, H., and Rajewsky, K. 1998. Somatic hypermutation in the heavy chain locus correlates with transcription. Immunity 9: 105-114.

Goodman, M.F. and Tippin, B. 2000. Sloppier copier DNA polymerases involved in genome repair. Curr. Opin. Genet. Dev. 10: $162-168$.

Gordon, M.S., Kanegai, C.M., Doerr, J.R., and Wall, R. 2003. Somatic hypermutation of the B cell receptor genes B29 $(\operatorname{Ig} \beta$, CD79b) and mb1 (Ig $\alpha$, CD79a). Proc. Natl. Acad. Sci. 100: 4126-4131.

Harris, R.S., Sale, J.E., Petersen-Mahrt, S.K., and Neuberger, M.S. 2002. AID is essential for immunoglobulin V gene conversion in a cultured B cell line. Curr. Biol. 12: 435-438.

Imai, K., Slupphaug, G., Lee, W.I., Revy, P., Nonoyama, S., Catalan, N., Yel, L., Forveille, M., Kavli, B., Krokan, H.E., et al. 2003. Human uracil-DNA glycosylase deficiency associated with profoundly impaired immunoglobulin class-switch recombination. Nat. Immunol. 4: 1023-1028.

Kenter, A.L. 2003. Class-switch recombination: After the dawn of AID. Curr. Opin. Immunol. 15: 190-198.

Kinoshita, K. and Honjo, T. 2001. Linking class-switch recombination with somatic hypermutation. Nat. Rev. Mol. Cell. Biol. 2: 493-503.

Kolodner, R. 1996. Biochemistry and genetics of eukaryotic mismatch repair. Genes \& Dev. 10: 1433-1442.

Li, Z., Woo, C.J., and Scharff, M.D. 2003. Mutations in AID and UNG extend the function of AID. Nat. Immunol. 4: 945946.

Lin, M.M., Green, N.S., Zhang, W., and Scharff, M.D. 1998. The effects of Em, 3'a (hs 1,2) and 3'k enhancers on mutation of an Ig-VDJ-Cg2a immunoglobulin heavy gene in cultured B cells. Int. Immunol. 10: 1121-1129.

MacLennan, I.C. 1994. Germinal centers. Ann. Rev. Immunol. 12: $117-139$.

Manis, J.P., Dudley, D., Kaylor, L., and Alt, F.W. 2002a. IgH class switch recombination to IgG1 in DNA-PKcs-deficient B cells. Immunity 16: 607-617.

Manis, J.P., Tian, M., and Alt, F.W. 2002b. Mechanism and control of class-switch recombination. Trends Immunol. 23: 31-39.

Martin, A. and Scharff, M.D. 2002a. AID and mismatch repair in antibody diversification. Nat. Rev. Immunol. 2: 605-614.

- 2002b. Somatic hypermutation of the AID transgene in B and non-B cells. Proc. Natl. Acad. Sci. 99: 1230412308.

Martin, A., Bardwell, P.D., Woo, C.J., Fan, M., Shulman, M.J., and Scharff, M.D. 2002. Activation-induced cytidine deaminase turns on somatic hypermutation in hybridomas. $\mathrm{Na}$ ture 415: 802-806.

Martin, A., Li, Z., Lin, D., Bardwell, P.D., Iglesias-Ussel, M.D., Edelmann, W., and Scharff, M.D. 2003. Msh2 ATPase activity is essential for somatic hypermutation at A-T basepairs and for efficient class switch recombination. J. Exp. Med. 198: $1171-1178$.

McDonald, J.P., Frank, E.G., Plosky, B.S., Rogozin, I.B., Masutani, C., Hanaoka, F., Woodgate, R., and Gearhart, P.J. 2003. 129-derived strains of mice are deficient in DNA polymerase $\iota$ and have normal immunoglobulin hypermutation. J. Exp. Med. 198: 635-643.

Michael, N., Shen, H.M., Longerich, S., Kim, N., Longacre, A., and Storb, U. 2003. The E box motif CAGGTG enhances somatic hypermutation without enhancing transcription. Immunity 19: 235-242.

Milstein, C., Neuberger, M.S., and Staden, R. 1998. Both DNA strands of antibody genes are hypermutation targets. Proc. Nat1. Acad. Sci. 95: 8791-8794.

Morvan, C.L., Pinaud, E., Decourt, C., Cuvillier, A., and Cogne, M. 2003. The immunoglobulin heavy-chain locus hs3b and hs4 3' enhancers are dispensable for VDJ assembly and somatic hypermutation. Blood 102: 1421-1427.

Mostoslavsky, R., Alt, F.W., and Bassing, C.H. 2003. Chromatin dynamics and locus accessibility in the immune system. Nat. Immunol. 4: 603-606.

Muramatsu, M., Sankaranand, V.S., Anant, S., Sugai, M., Kinoshita, K., Davidson, N.O., and Honjo, T. 1999. Specific expression of activation-induced cytidine deaminase (AID), a novel member of the RNA-editing deaminase family in germinal center B cells. J. Biol. Chem. 274: 1847018476.

Muramatsu, M., Kinoshita, K., Fagarasan, S., Yamada, S., Shinkai, Y., and Honjo, T. 2000. Class switch recombination and hypermutation require activation-induced cytidine deaminase (AID), a potential RNA editing enzyme. Cell 102: 553-563.

Navaratnam, N., Morrison, J.R., Bhattacharya, S., Patel, D., Funahashi, T., Giannoni, F., Teng, B.B., Davidson, N.O., and Scott, J. 1993. The p27 catalytic subunit of the apolipoprotein B mRNA editing enzyme is a cytidine deaminase. $I$. Biol. Chem. 268: 20709-20712.

Papavasiliou, F.N. and Schatz, D.G. 2002. Somatic hypermutation of immunoglobulin genes: Merging mechanisms for genetic diversity. Cell 109(Suppl): S35-S44.

Pasqualucci, L., Migliazza, A., Fracchiolla, N., William, C., Neri, A., Baldini, L., Chaganti, R.S.K., Klein, U., Kuppers, R., Rajewsky, K., et al. 1998. BCL-6 mutations in normal germinal center B cells: Evidence of somatic hypermutation 
acting outside Ig loci. Proc. Natl. Acad. Sci. 95: 1181611821 .

Pasqualucci, L., Neumeister, P., Goossens, T., Nanjangud, G., Chaganti, R.S., Kuppers, R., and Dalla-Favera, R. 2001. Hypermutation of multiple proto-oncogenes in B-cell diffuse large-cell lymphomas. Nature 412: 341-346.

Peters, A. and Storb, U. 1996. Somatic hypermutation of immunoglobulin genes is linked to transcription initiation. Immunity 4: 57-65.

Petersen-Mahrt, S.K., Harris, R.S., and Neuberger, M.S. 2002. AID mutates E. coli suggesting a DNA deamination mechanism for antibody diversification. Nature 418: 99-103.

Pham, P., Bransteitter, R., Petruska, J., and Goodman, M.F. 2003. Processive AID-catalysed cytosine deamination on single-stranded DNA simulates somatic hypermutation. $\mathrm{Na}$ ture 424: 103-107.

Poltoratsky, V., Goodman, M.F., and Scharff, M.D. 2000. Error prone candidates vie for somatic mutation. I. Exp. Med. 192: F27-F30.

Rada, C. and Milstein, C. 2001. The intrinsic hypermutability of antibody heavy and light chain genes decays exponentially. EMBO J. 20: 4570-4576.

Rada, C., Ehrenstein, M.R., Neuberger, M.S., and Milstein, C. 1998. Hot spot focusing of somatic hypermutation in MSH2deficient mice suggests two stages of mutational targeting. Immunity 9: 135-141.

Rada, C., Williams, G.T., Nilsen, H., Barnes, D.E., Lindahl, T., and Neuberger, M.S. 2002. Immunoglobulin isotype switching is inhibited and somatic hypermutation perturbed in UNG-deficient mice. Curr. Biol. 12: 1748-1755.

Rajewsky, K., Forster, I., and Cumano, A. 1987. Evolutionary and somatic selection of the antibody repertoire in the mouse. Science 238: 1088-1094.

Ramiro, A.R., Stavropoulos, P., Jankovic, M., and Nussenzweig, M.C. 2003. Transcription enhances AID-mediated cytidine deamination by exposing single-stranded DNA on the nontemplate strand. Nat. Immunol. 4: 452-456.

Reina-San-Martin, B., Difilippantonio, S., Hanitsch, L., Masilamani, R.F., Nussenzweig, A., and Nussenzweig, M.C. 2003. $\mathrm{H} 2 \mathrm{AX}$ is required for recombination between immunoglobulin switch regions but not for intra-switch region recombination or somatic hypermutation. J. Exp. Med. 197: 17671778 .

Revy, P., Muto, T., Levy, Y., Geissmann, F., Plebani, A., Sanal, O., Catalan, N., Forveille, M., Dufourcq-Labelouse, R., Gennery, A., et al. 2000. Activation-induced cytidine deaminase (AID) deficiency causes the autosomal recessive form of the hyper-IgM syndrome (HIGM2). Cell 102: 565575.

Rogozin, I.B. and Kolchanov, N.A. 1992. Somatic hypermutagenesis in immunoglobulin genes, II: Influence of neighbouring base sequences on mutagenesis. Biochim. Biophy. Acta 1171: $11-18$.

Sale, J.E. and Neuberger, M.S. 1998. TdT-accessible breaks are scattered over the immunoglobulin V domain in a constitutively hypermutating B cell line. Immunity 9: 859-869.

Schlissel, M.S. 2000. Perspectives: transcription: A tail of histone acetylation and DNA recombination. Science 287: 438440.

Schrader, C.E., Edelmann, W., Kucherlapati, R., and Stavnezer, J. 1999. Reduced isotype switching in splenic B cells from mice deficient in mismatch repair enzymes. J. Exp. Med. 190: 323-330.

Schrader, C.E., Vardo, J., and Stavnezer, J. 2002. Role for mismatch repair proteins Msh2, Mlh1, and Pms2 in immuno- globulin class switching shown by sequence analysis of recombination junctions. J. Exp. Med. 195: 367-373.

- 2003. Mlh1 can function in Antibody class switch recombination independently of Msh2. J. Exp. Med. 197: 13771383.

Sharpe, M.J., Neuberger, M., Pannell, R., Surani, M.A., and Milstein, C. 1990. Lack of somatic mutation in a kappa light chain transgene. Eur. J. Immunol. 20: 1379-1385.

Shen, H.M., Peters, A., Baron, B., Zhu, X., and Storb, U. 1998. Mutation of BCL- 6 gene in normal B cells by the process of somatic hypermutation of Ig genes. Science 280: 1750-1752.

Shen, H.M., Michael, N., Kim, N., and Storb, U. 2000. The TATA binding protein, c-Myc and survivin genes are not somatically hypermutated, while Ig and BCL6 genes are hypermutated in human memory B cells. Int. Immunol. 12: 1085-1093.

Shinkura, R., Tian, M., Smith, M., Chua, K., Fujiwara, Y., and Alt, F.W. 2003. The influence of transcriptional orientation on endogenous switch region function. Nat. Immunol. 4: 435-441.

Sohail, A., Klapacz, J., Samaranayake, M., Ullah, A., and Bhagwat, A.S. 2003. Human activation-induced cytidine deaminase causes transcription-dependent, strand-biased C to U deaminations. Nucleic Acids Res. 31: 2990-2994.

Stavnezer, J. 1996. Antibody class switching. Adv. Immunol. 61: 79-146.

2000. Molecular processes that regulate class switching. Curr. Top. Microbiol. Immunol. 245: 127-168.

Ta, V.T., Nagaoka, H., Catalan, N., Durandy, A., Fischer, A., Imai, K., Nonoyama, S., Tashiro, J., Ikegawa, M., Ito, S., et al. 2003. AID mutant analyses indicate requirement for classswitch-specific cofactors. Nat. Immunol. 4: 843-848.

Tonegawa, S. 1983. Somatic generation of antibody diversity. Nature 302: 575-581.

Tumas-Brundage, K. and Manser, T. 1997. The transcriptional promoter regulates hypermutation of the antibody heavy chain locus. J. Exp. Med. 185: 239-250.

Tumas-Brundage, K., Vora, K.A., Giusti, A.M., and Manser, T. 1996. Characterization of the cis-acting elements required for somatic hypermutation of murine antibody $\mathrm{V}$ genes using conventional transgenic and transgene homologous recombination approaches. Semin. Immunol. 8: 141-150.

Tumas-Brundage, K.M., Vora, K.A., and Manser, T. 1997. Evaluation of the role of the $3^{\prime} \alpha$ heavy chain enhancer $\left[3^{\prime} \alpha\right.$ $\mathrm{E}(\mathrm{hs} 1,2)]$ in $\mathrm{Vh}$ gene somatic hypermutation. Mol. Immunol. 34: 367-378.

Vora, K.A., Tumas-Brundage, K.M., Lentz, V.M., Cranston, A., Fishel, R., and Manser, T. 1999. Severe attenuation of the B cell immune response in Msh2-deficient mice. J. Exp. Med. 189: 471-481.

Wei, K., Kucherlapati, R., and Edelmann, W. 2002. Mouse models for human DNA mismatch-repair gene defects. Trends Mol. Med. 8: 346-353.

Weill, J.C. and Reynaud, C.A. 1996. Rearrangement/hypermutation/gene conversion: When, where and why? Immunol. Today 17: 92-97.

Woo, C.J., Martin, A., and Scharff, M.D. 2003. Induction of hypermutation is associated with modifications of variable region chromatin in BL2 cells. Immunity 19: 479-489.

Yelamos, J., Klix, N., Goyenechea, B., Lozano, F., Chui, Y.L., Gonzalez Fernandez, A., Pannell, R., Neuberger, M.S., and Milstein, C. 1995. Targeting of non-Ig sequences in place of the V segment by somatic hypermutation. Nature 376: 225 229.

Yoshikawa, K., Okazaki, I.M., Eto, T., Kinoshita, K., Muramatsu, M., Nagaoka, H., and Honjo, T. 2002. AID enzyme- 
induced hypermutation in an actively transcribed gene in fibroblasts. Science 296: 2033-2036.

Yu, K., Chedin, F., Hsieh, C.L., Wilson, T.E., and Lieber, M.R. 2003. R-loops at immunoglobulin class switch regions in the chromosomes of stimulated B cells. Nat. Immunol. 4: 442451.

Zan, H., Komori, A., Li, Z., Cerrutti, M., Flajnik, M.F., Diaz, M., and Casali, P. 2001. The translesional polymerase $\zeta$ plays a major role in Ig and Bcl-6 somatic mutation. Immunity 14: 643-653.

Zan, H., Wu, X., Komori, A., Holloman, W.K., and Casali, P. 2003. AID-dependent generation of resected double-strand DNA breaks and recruitment of $\operatorname{Rad} 52 / \operatorname{Rad} 51$ in somatic hypermutation. Immunity 18: 727-738.

Zeng, X., Winter, D.B., Kasmer, C., Kraemer, K.H., Lehmann, A.R., and Gearhart, P.J. 2001. DNA polymerase $\eta$ is an A-T mutator in somatic hypermutation of immunoglobulin variable genes. Nat. Immunol. 2: 537-541. 


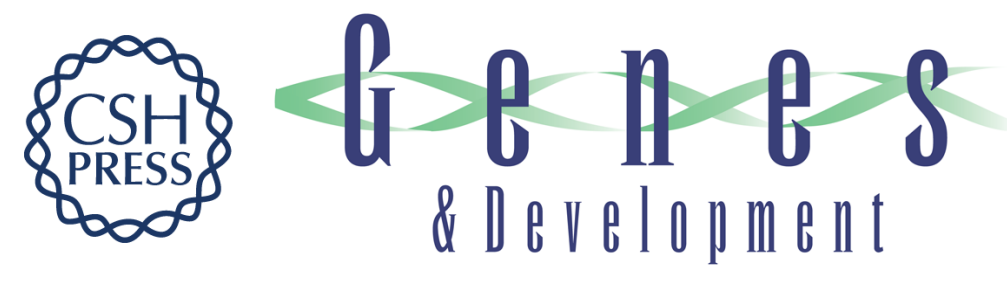

\section{The generation of antibody diversity through somatic hypermutation and class switch recombination}

Ziqiang Li, Caroline J. Woo, Maria D. Iglesias-Ussel, et al.

Genes Dev. 2004, 18:

Access the most recent version at doi:10.1101/gad.1161904

$\begin{array}{ll}\text { References } & \begin{array}{l}\text { This article cites } 85 \text { articles, } 31 \text { of which can be accessed free at: } \\ \text { http://genesdev.cshlp.org/content/18/1/1.full.html\#ref-list-1 }\end{array}\end{array}$

License

Email Alerting Receive free email alerts when new articles cite this article - sign up in the box at the top Service right corner of the article or click here.

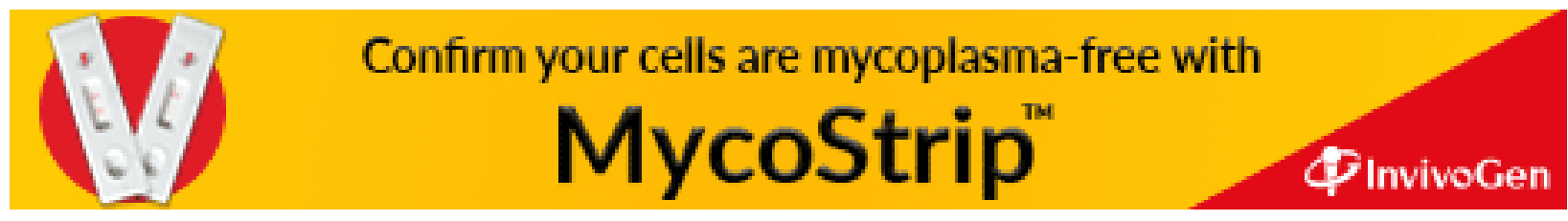

